\title{
Repetition redundancy and visual form discrimination'
}

G. R. LOCKHEAD

DUKE UNIVERSITY

\begin{abstract}
Abstraet
An experiment by Eriksen and Lappin was partially replicated with the addition of a control condition. The results demonstrate that a theoretical model proposed by those authors is not correct and that simultaneously repeating a visual form in different areal portions of the retina leads to improved discrimination performance only when Ss are operating under position uncertainty, i.e., when they do not know where the stimulus will appear.
\end{abstract}

\section{Introduction}

A recent paper by Eriksen \& Lappin (1965) presented a theoretical model, and supporting data, which purportedly show that the simultaneous repetition of the same form in a tachistoscopic situation leads to an increase in the probability that $S$ will correctly report that form. It is the purpose of this note to show that their interpretation of their data is not correct and to provide an alternative interpretation.

The basis of Eriksen and Lappin's model, perhaps stated too simply here, is that neurons and ganglia in the retina show spontaneous discharge, noise, and that this noise is uncorrelated in different areal portions of the retina. If different retinal areas are stimulated simultaneously with the identical stimulus, according to the model, identification accuracy of the stimulus should be superior to the stimulation of only one retinal area since the noise in these two areas is uncorrelated as long as the separation between elements is large enough. I am not taking exception to the argument of uncorrelated spontaneous neural discharges. I will attempt to show, however, that the model is incorrect as an interpretation of their data.

In their experiment designed to test this model, Ss were presented the capital letters $\mathrm{A}, \mathrm{T}$, and $\mathrm{U}$ tachistoscopically and asked to report which letter was presented on a given trial. The exposure durations were determined by each S's threshold, and presented letters were spaced around an imaginary circle, with radii of $1 / 2^{\circ}, 1^{\circ}$, and $2^{\circ}$ in three separately run conditions. Spacing was not found to be important. The conditions within a given spatial separation condition were the presentation of any of the three letters where each letter could be repeated $0,1,3$, or 5 times. There were six positions around the imaginary circle, with fixation in the center, in which these stimuli could occur. Thus, on some trials a $\mathrm{S}$ would be presented a single $\mathrm{A}$ in one of the locations. On other trials, where all trials were randomly intermixed, two, four, or six As would be simultaneously presented in some or all of these positions. Similarly for the Ts and Us. Ss made forced-choice judgments as to which letter had been presented and also gave a three category confidence judgment of their response. In this experiment, the number of form repetitions was a highly significant source of variance and the authors report that repetition redundancy (the repeating of the same stimulus) improves discrimination performance and that their "model provides a satisfactory fit for the observed data points."

In that experiment, Ss were presented all conditions of both redundancy and no redundancy within each experimental situation. Such methodology is good experimental design for reducing differential variance due to daily $S$, or environmental, or what have you, effects. However, in this situation, it also meant that Ss never knew, on each stimulus presentation, whether they would be presented with $1,2,4$, or 6 letters and they also never knew where the letters would occur in which of the six possible positions. Consider how you might expect $S$ to perform in a discrimination situation in which on one trial he might be presented with a letter on either the left of the visual field or on the right, or on both left and right simultaneously. It is inconceivable, if the letters are spread far enough apart, that presentation of the second letter could impair his discrimination on the first letter; but it is conceivable that the repetition of the letter could improve performance over the single letter, since $\mathrm{S}$ would be able to attend to the appropriate stimulus location no matter where it was. That is, repetition redundancy must be effective when the $S$ is operating under position uncertainty - when he doesn't know where the stimulus will occur. This is what Eriksen and Lappin have shown. They have not, however, shown that the neural elements on the retina are functionally independent, each providing an element of discrimination along with a confidence judgment as they suggest.

I am arguing that this improvement in discrimination performance due to repetition redundancy, reported in the Eriksen and Lappin study, is due to position uncertainty and not to uncorrelated noise in different neural elements. This argument was tested by a partial replication of one of Eriksen and Lappin's conditions when position uncertainty both was and was not a variable. 


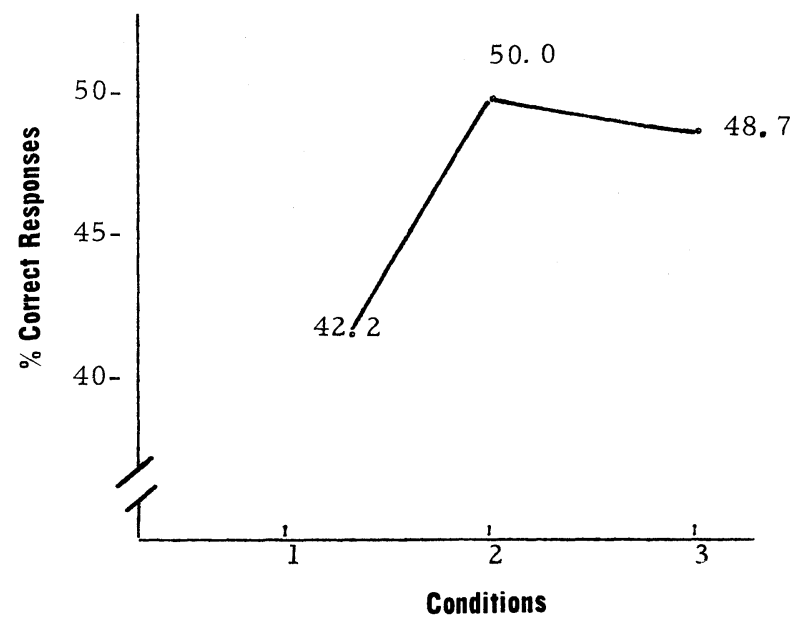

Fig. 1. Percent correct identifications for the conditions (1) Single letter position unknown, (2) single letter position known, and (3) two letters. Data are means of $\mathbf{1 8 0 0}$ observations for each point.

\section{Method}

The form stimuli were the capital letters $A, T$, and $\mathrm{U}$, black on white and subtending $0.2^{\circ}$ of visual angle. They were presented in a tachistoscope with a dim white adaptation field and a black fixation point. The retinal spacing used was $2^{\mathrm{O}}$. An element could appear either at the $20^{\prime}$ clock or $100^{\prime}$ clock position on an imaginary circle around the fixation point. (Eriksen and Lappin had $0.3^{\circ}$ stimuli, a black adaptation field with a bright fixation point, and these same positions and retinal spacings as well as many others; according to theory these slight procedural differences should be trivial.) During two practice sessions, an exposure duration was determined for each $\mathrm{S}$ that resulted in approximately 50\% identification accuracy for a single presentation of a form. The resulting durations were all approximately $20 \mathrm{msec}$. There were three experimental sessions. The order in which the conditions were presented was partially counterbalanced between Ss and sessions, and each $\mathrm{S}$ gave 150 responses to each condition during each session. The conditions were: (1) the single letters presented randomly at either the $100^{\prime}$ clock position or the $20^{\prime}$ clock position but not both, this condition provides position uncertainty; (2) the single letters presented randomly at the 10 o'clock position only, there was no position uncertainty; and (3) the letters presented in both the 10 o'clock and 2 o'clock positions simultaneously, this condition provides repetition redundancy without position uncertainty. Stimulus presentations were selfpaced and Ss were told the correct response immediately after responding. The Ss were four paid males, two practiced and two not practiced in discrimination experiment situations.

\section{Results and Discussion}

The data reported are the present correct responses pooled over Ss and are presented in Fig. 1. Analysis of variance showed a significant effect of treatment conditions $(p<.01)$. All Ss showed the same ordering of conditions, the single element with position known was best, the double element condition next best, and the single element with position unknown worst. The difference between the first two conditions is not statistically reliable.

According to Eriksen and Lappin's model, discrimination performance on both the single element conditions should be the same and less than performance when there is repetition redundancy. According to arguments concerning position uncertainty, performance should be the same for the single and double element conditions when Ss knew where the stimuli would appear, provided the repeated element did not interfere with perception, and both of these conditions should be superior to a single element presentation when there is position uncertainty. The results of the experiment are consonant with this second position.

\section{Reference}

Eriksen. C. W . \& Lappin. J S Internal perceptual system noise and redundancy in simultaneous inputs in form identification. Psuchon. Sci., 1965, 2, 351-352

\section{Note}

1 This study was supported by Public Health Service research grant MH 11769-01

For Reply by C. W. Eriksen and J. S. Lappin see page 366. 